\title{
BI-ARTINIAN NOETHERIAN RINGS
}

\author{
E. A. WHELAN \\ Department of Mathematics, London School of Economics, Houghton Street, \\ London WC2A $2 A E$, United Kingdom \\ e-mail:e.a.whelan@lse.ac.uk
}

(Received 23 February, 1999)

\begin{abstract}
A noetherian ring $R$ satisfies the descending chain condition on twosided ideals ("is bi-artinian") if and only if, for each prime $P \in \operatorname{spec}(R), R / P$ has a unique minimal ideal (necessarily idempotent and left-right essential in $R / P$ ). The analogous statement for merely right noetherian rings is false, although our proof does not use the full noetherian condition on both sides, requiring only that twosided ideals be finitely generated on both sides and that $R / Q$ be right Goldie for each $Q \in \operatorname{spec}(R)$. Examples exist, for each $n \in \mathbb{N}$ and in all characteristics, of bi-artinian noetherian domains $D_{n}$ with composition series of length $2^{n}$ and with a unique maximal ideal of height $n$. Noetherian rings which satisfy the related E-restricted bid.c.c. do not, in general, satisfy the second layer condition (on either side), but do satisfy the Jacobson conjecture.
\end{abstract}

1991 Mathematics Subject Classification. 16P, 16N.

1. Introduction. A ring $R$ (always associative with unity) is said to be bi-artinian if it satisfies the d.c.c. on (two-sided) ideals. For both commutative noetherian rings and so-called bi-noetherian polynormal (BPN) rings it is known that a ring in the specified class is bi-artinian if and only if every prime ideal is maximal (see [1, p. 90] and [19, p. 93] respectively). At the opposite extreme lie the endomorphism rings of large-enough dimensional vector spaces, which are always bi-artinian but need not be bi-noetherian [5, Ch. 8].

This note obtains necessary and sufficient conditions (Section 4) for a (left-right) noetherian ring to be bi-artinian, namely that $R$ is bi-artinian if and only if, for every $P \in \operatorname{spec}(R), R / P$ has a simple idempotent ideal. Following [20] we say that (in any ring) a prime with that property is quasi-primitive. It is known [19, p. 97] that, in commutative or BPN rings, quasi-primitive ideals are necessarily maximal, so this result extends or parallels the known results for those two cases. On the other hand, the analogous statement for right noetherian rings is false (Example 3.8).

In fact, our results apply to a larger class of rings than the noetherian: we require only that $t w o$-sided ideals of a ring $R$ be finitely generated on each side and that $R / Q$ be right Goldie for each $Q \in \operatorname{spec}(R)$. In the noetherian case, our main result is an easy (though, as far as we know, an unremarked) consequence of an old result of Jategaonkar: see Remark 7.5(A).

Other results of the paper concern direct sum decompositions (Section 5) and noetherian rings which satisfy the restricted bi-d.c.c. (Section 6), including (Remark 6.4(B) and Example 6.5) a class of noetherian rings which, in general, fail the second layer condition on both sides but which satisfy a strong form of the Jacobson Conjecture (Corollary 6.3). Sections 2 and 3 contain technicalities, and Section 7 covers examples and applications, including (Examples 7.2(E) and 7.2(F)), for any integer $n \in \mathbb{N}$ and any characteristic, a prime noetherian ring $W_{n}$ (that can be chosen to be a 
domain) which has a bimodule composition series of length $2^{n}$ and a unique maximal ideal of height $n$.

As noted, almost all our results use the full noetherian condition on neither side; indeed right but not left noetherian rings meeting the required conditions are readily constructed (Remark 4.3(D)). We also note that, for some noetherian rings $R$ which are not bi-artinian, there is a (right) denominator set $\mathrm{D} \subseteq R$ such that $Q=R \mathrm{D}^{-1}$ is a bi-artinian ring, and then, for a broad range of bimodules ${ }_{S} M_{R}$, the related bimodule ${ }_{S}\left(M \otimes_{R} Q\right)_{Q}$ has a bimodule composition series. We believe this "localisation" technique may be fairly widely applicable in the study of noetherian bimodules, particularly when "traditional" localisation, in which it is expected of a local ring $S$ that $S / J(S)$ should be artinian, is unavailable: see Remark 7.4(B) and [21].

Thanks are due to Dr. A. W. Chatters and to Professors K. A. Brown and J. C. Robson for encouraging my interest in noetherian rings.

2. Notation and terminology. All undefined terminology and notation are standard, and in particular follow [6], but the following points are worth noting. The symbols $\leq,<$ and $\leq_{e}$ respectively denote inclusion, strict inclusion and (bi-)essential inclusion of (bi)modules or one- or two-sided ideals. The term ideal (unqualified) always means a two-sided ideal, the ideal lattice of $R$ is denoted by $\mathrm{I}(R)$, and the sub-lattice of bi-essential ideals by $\mathrm{E}(R)$. The prefix $b i$ - denotes reference to a system of bimodules or of ideals, e.g. bi-essential means essential as a sub-bimodule. When a one-sided property holds on both sides we drop references to sides, e.g. a bimodule is noetherian if it is left-right noetherian. Following [23] a bimodule is right (left) semi-noetherian if every sub-bimodule is right (left) finitely generated. The rank of a (bi-)module $M_{R}$ or ${ }_{S} M_{R}$ is its uniform dimension as (bi-)module, and is denoted by $\operatorname{rank}\left(M_{R}\right)$ or $\operatorname{rank}\left({ }_{S} M_{R}\right)$.

The heart of a ring or non-zero bimodule is the intersection of all its non-zero ideals or sub-bimodules, and is denoted by the function $\mathrm{H}(-)$. In a quasi-primitive ring $R, H=\mathrm{H}(R)$ is the unique minimal non-zero ideal of $R$ and is idempotent. The set of quasi-primitive ideals of a ring $S$ is denoted by quas $(S)$, and the intersection of its elements is the anti-simple radical of $S$, denoted by $\mathrm{A}(S)$ (see [17, pp. $74 \mathrm{ff}$.$] ). The$ sets of minimal prime and of maximal ideals of $S$ are denoted by $\min (S)$ and $\max (S)$ respectively. Evidently $\max (S) \subseteq$ quas $(S)$, so $\mathrm{A}(S) \subseteq \mathrm{B}(S) \bigcap_{M \in \max (S)} M$, the BrownMcCoy radical of S. The prime and the Jacobson radicals of $S$ are denoted by $\mathrm{P}(S)$ and $\mathrm{J}(S)$ respectively. The bi-socle of a ring or bimodule is the intersection of all its bi-essential sub-objects, i.e. the sum of all its simple sub-objects (see [4, p. 3] or [6, p. 59]).

We use without explicit mention six standard facts.

(1) If $M={ }_{S} M_{R}$ is a left semi-noetherian right faithful bimodule then $R_{R}$ embeds in a finite direct sum of copies of $M_{R}$, so that if (for example) $\operatorname{rank}\left(M_{R}\right)<\infty$ then $\operatorname{rank}\left(R_{R}\right)<\infty$.

(2) If $0 \neq I \in \mathrm{I}(R)$ and $R$ is prime then $I \leq_{e} R$ and $I \leq_{e} R_{R}$.

(3) If $I \in \mathrm{I}(R)$ and $R$ is bi-noetherian then there are finitely many primes minimal over $I$, say $P_{1}, \ldots, P_{r}$, and if $N=P_{1} \cap \ldots \cap P_{r}$ then there exist $n \in \mathbb{N}$ and (not necessarily distinct) $Q_{1}, \ldots, Q_{n} \in\left\{P_{1}, \ldots, P_{r}\right\}$ such that $N^{n} \leq Q_{1} Q_{2} \ldots Q_{n} \leq I \leq N$ (with each $P_{j}$ occurring at least once among $Q_{1}, \ldots, Q_{n}$ ). 
(4) If $L=L_{R} \leq_{e} M=M_{R}$ and $m \in M$ then $\{r \in R: m r \in L\} \leq_{e} R_{R}$.

(5) If $R$ is semiprime and $\operatorname{rank}\left({ }_{R} R_{R}\right)=n<\infty$ then $\operatorname{card}(\min (R))=n$, and $I \in \mathrm{E}(R)$ if and only if $I \not \leq P$ for all $P \in \min (R)$.

(6) If $R$ is semiprime and $I \in \mathrm{I}(R)$, and if $J \in \mathrm{I}(R)$ is maximal with respect to $I \cap J=0$, then $J$ is unique, and $\operatorname{ann}\left({ }_{R} I\right)=\operatorname{ann}\left(I_{R}\right)=J$.

3. Technical Lemmas. The following is closely related to [6, Lemma 7.3].

Lemma 3.1. Let $M={ }_{S} M_{R}$ be left semi-noetherian, such that: $R_{R}$

(a) if $N={ }_{S} N_{R} \leq{ }_{S} M_{R}$ and $N_{R} \leq{ }_{e} L=L_{R} \leq M_{R}$, then $\mathrm{I}(R) \ni \operatorname{ann}\left((S L / N)_{R}\right) \leq_{e}$

(b) if $K={ }_{S} K_{R} \leq{ }_{S} M_{R}$ and $K_{R} \leq{ }_{e} M_{R}$, then there exists $\mathrm{I}(R) \ni J \leq{ }_{e} R_{R}$ such that $M J \leq K$.

Proof. (after [6, 7]). (a) Let $S L=S x_{1}+\ldots+S x_{m}$; each $x_{i}$ is a finite left $S$-linear combination of elements of $L$, and we can therefore assume each $x_{i} \in L$. For each $i$ there exists $E_{i} \leq_{e} R_{R}$ such that $x_{i} E \leq N$; let $E=\cap E_{i} \leq_{e} R_{R}$. Then $(S L)(R E)=$ $S L E=S x_{1} E+\ldots+S x_{m} E \leq N$ and $\mathrm{I}(R) \ni R E \leq_{e} \operatorname{ann}\left((S L / N)_{R}\right) \leq_{e} R_{R}$. Part (b) follows by similar arguments.

We recall the well known fact that a maximal annihilator of a non-zero sub(-bi)module is a prime ideal. If $0 \neq{ }_{S} M_{R}$ let $B$-spec $\left(M_{R}\right)$ be the set of maximal annihilators of non-zero sub-bimodules of the bimodules ${ }_{S}(M / N)_{R}$ for bimodules $N$ such that ${ }_{S} N_{R}<{ }_{S} M_{R}$, and note that if $R$ is bi-noetherian then $B-\operatorname{spec}\left(M_{R}\right) \neq \varnothing$.

Lemma 3.2. (a) Let $M={ }_{S} M_{T}$ be semi-noetherian; if $T$ is bi-noetherian then $\operatorname{rank}\left((M / N)_{T}\right)<\infty$ for every $N={ }_{S} N_{T}<{ }_{S} M_{T}$ if and only if $\operatorname{rank}\left((T / P)_{T}\right)<\infty$ for every $P \in B-\operatorname{spec}\left(M_{T}\right)$.

(b) A left semi-noetherian semiprime ring $W$ is right non-singular.

(c) A semi-noetherian ring $R$ is fully right Goldie if and only if $\operatorname{rank}\left((R / I)_{R}\right)<\infty$ for every $I \in \mathrm{I}(R)$.

Proof. (a) In the circumstances of (a), suppose that $\operatorname{rank}\left((M / N)_{T}\right)<\infty$ for every $N={ }_{S} N_{T}<{ }_{S} M_{T}$. If $P \in B$-spec $(M R)$ then $(T / P)_{T}$ embeds in a finite direct sum of copies of (some non-zero sub-bimodule of) $(M / N)_{T}$, so $\operatorname{rank}\left((T / P)_{T}\right)<\infty$.

In the opposite direction, if our claim is false then by noetherian induction we can assume that $K={ }_{S} K_{T}<{ }_{S} M_{T}$ is maximal with respect to that failure. If $Q \in B$-spec $\left((M / N)_{T}\right)$ let $K<L={ }_{S} L_{T} \leq{ }_{S} M_{T}$ be such that $Q=\operatorname{ann}\left((L / K)_{T}\right)$, and then let $W$ be the right Goldie ring of quotients of $(T / Q)$. By Lemma $3.1(L / K)_{T / Q}$ is torsionfree, and hence $(L / K)_{T / Q} \rightarrow\left((L / K) \otimes_{T / Q} W\right)_{T / Q}$ is injective. Also $(L / K)_{T / Q}$ is finitely generated, and hence so is $\left((L / K) \otimes_{T / Q} W\right)_{W}$. Since $W$ is artinian semi-simple, so is $\left((L / K) \otimes_{T / Q} W\right)_{W}$, from which, by standard arguments, $\operatorname{rank}\left([(L / K) \otimes W]_{W}\right)=\operatorname{rank}\left((L / K)_{T / Q}\right)=\operatorname{rank}\left((L / K)_{T}\right)<\infty$.

Now by Zorn's Lemma let $K \leq C=C_{T} \leq M_{T}$ be maximal with respect to $C \cap L=K$; then $((C+L) / L)_{T} \cong(C /(C \cap L))_{T}=(C / K)_{T}$ so $(C / K)_{T}$ embeds in $(M / L)_{T}$, and by the maximality of $K, \operatorname{rank}\left((M / L)_{T}\right)<\infty$, so $\operatorname{rank}\left((C / K)_{T}\right)<\infty$. But $((C / K) \oplus(L / K))_{T} \leq_{e}(M / K)_{T}$ by the maximality of $\mathrm{C}$, so $\operatorname{rank}\left((M / K)_{T}\right)=$ $\operatorname{rank}\left([(C / K) \oplus(L / K)]_{T}\right)=\operatorname{rank}\left((C / K)_{T}\right)+\operatorname{rank}\left((L / K)_{T}\right)<\infty ;$ contradiction. 
(b) Suppose that $W$ is left semi-noetherian semiprime and that $Z$ is its right singular ideal. As in the proof of Lemma 3.1, ann $\left(Z_{W}\right) \leq_{e} W_{W}$; if $0 \neq Z$ then $J=Z \cap \operatorname{ann}\left(Z_{W}\right) \neq 0$. But $J^{2}=0$ and $W$ is semiprime, so we must have $J=Z=0$.

(c) One direction is covered by (b). In the other, if $P \in \operatorname{spec}(R)$ then $R / P$ is right non-singular, by (b), and, by a result of Gabriel [6, Theorem 4.28, p. 82] plus [6, Chapter 5], $R / P$ is right Goldie (using $\left.\operatorname{rank}\left((R / P)_{R}\right)<\infty\right)$.

Lemma 3.2 will allow us to assume (see Lemma 3.4) that, for the bimodules ${ }_{S} M_{R}$ in which we shall be interested, $\operatorname{rank}\left((M / N)_{R}\right)<\infty$ for every $N={ }_{S} N_{R} \leq{ }_{S} M_{R}$. We shall refer to bimodules with that property as being fully of finite right rank.

Suppose that $R$ is a bi-uniform ring, and let $x \in M=M_{R}$. Then $x$ is said to be an $\mathrm{I}(R)$-torsion element if $x I=0$ for some $0 \neq I \in \mathrm{I}(R)$. The set of $\mathrm{I}(R)$-torsion elements forms a submodule $Y^{*}(M)$, which is a sub-bimodule if $M$ is a bimodule. A module $M$ is $\mathrm{I}(R)$-torsionfree if $Y^{*}(M)=0$, and if $0 \neq M_{R}$ is $\mathrm{I}(R)$-torsionfree then $R$ is clearly prime. This terminology follows [20]: other terms for the same concept are prime module and fully faithful module (see [6, p. 31] or [11, p. 107]). A non-zero $\mathrm{I}(R)$-torsionfree module is, a fortiori, faithful.

The dual concept to a module being $\mathrm{I}(R)$-torsionfree is it being $\mathrm{I}(R)$-anti-torsion [20]. A module $M=M_{R}$ is said to be $\mathrm{I}(R)$-anti-torsion if $M=M I$ for all $0 \neq I \in \mathrm{I}(R)$. If $0 \neq M$ is $\mathrm{I}(R)$-anti-torsion then $R$ is necessarily prime, and (see [20]) any module $M_{R}$ over a prime ring $R$ has a unique maximal submodule $Z^{*}(M)$ which is $\mathrm{I}(R)$-antitorsion. For example any faithful simple (right) module over a (right primitive) ring $R$ is (right) $\mathrm{I}(R)$-anti-torsion and $\mathrm{I}(R)$-torsionfree, while the metacyclic $\mathbb{Z}$-module $\mathbb{Z}_{P}^{\infty}, p>0$ a rational prime, is simultaneously $\mathrm{I}(\mathbb{Z})$-torsion and $\mathrm{I}(\mathbb{Z})$-anti-torsion.

Let $R$ be a prime ring: it was noted in [20] that the duality between the concepts $\mathrm{I}(R)$-anti-torsion and $\mathrm{I}(R)$-torsion-free lies in the fact that $0 \neq M=M_{R}$ is $\mathrm{I}(R)$-antitorsion if and only if every non-zero factor module is faithful, and $0 \neq M_{R}$ is $\mathrm{I}(R)$ torsionfree if and only if every non-zero submodule is faithful. The following lemmas show that, in certain circumstances, the two concepts are also equivalent.

Lemma 3.3. Let $M=M_{R}$ or $M={ }_{S} M_{R}$, where $R$ is a prime ring. Then the following conditions on $M$ are equivalent:

(1) every sub(-bi)module $L \leq M$ is $\mathrm{I}(R)$-anti-torsion;

(2) every factor (bi)module $M / L$ where $L \leq M$ is $\mathrm{I}(R)$-torsionfree;

(3) every (bi)module $L / K$ is $\mathrm{I}(R)$-torsionfree, where $K \leq L \leq M$;

(4) every (bi)module $L / K$ is $\mathrm{I}(R)$-anti-torsion, where $K \leq L \leq M$.

Proof. Straightforward.

Lemma 3.4. Let $0 \neq M={ }_{S} M_{R}$ be semi-noetherian, fully of finite right rank, and right $\mathrm{I}(R)$-anti-torsion.

(1) Suppose that, for each $0 \neq N \leq{ }_{S} M_{R}$ there exists $0 \neq L={ }_{S} L_{R} \leq{ }_{S} N_{R}$ such that $L_{R}$ is $\mathrm{I}(R)$-anti-torsion. Then $M_{R}$ is $\mathrm{I}(R)$-torsionfree and ${ }_{S} M_{R}$ has bi-essential bisocle $\mathrm{BS}(M)$ which is $\mathrm{I}(R)$-anti-torsion.

(2) Suppose further that every $N={ }_{S} N_{R} \leq{ }_{S} M_{R}$ is right $\mathrm{I}(R)$-anti-torsion; then ${ }_{S} M_{R}$ has a composition series.

Proof. If the conditions of (1) are satisfied, suppose $0 \neq K={ }_{S} K_{R} \leq{ }_{S} M_{R}$ is $\mathrm{I}(R)$ torsion; without loss of generality we may suppose that $K_{R}$ is also $\mathrm{I}(R)$-anti-torsion, 
and that ${ }_{S} K=S p_{1}+\ldots+S p_{r}$. If $0 \neq A_{i} \in \mathrm{I}(R)$ are such that $p_{i} A_{i}=0$ for each $i$, let $0 \neq A=\cap A_{i} \in I(R)$ : then $K A=0$, contradicting the anti-torsion property. Hence $M_{R}$ is $\mathrm{I}(R)$-torsionfree.

Now use induction on $\operatorname{rank}\left(M_{R}\right)$. If $\operatorname{rank}\left(M_{R}\right)=1$ and $0 \neq E={ }_{S} E_{R} \leq M$ then $E_{R} \leq_{e} M_{R}$, so $(M / E)_{R}$ is unfaithful (Lemma 3.1). But every non-zero homomorphic image of an anti-torsion module is faithful [20, p. 618], so $E=M$, i.e. $M$ is a simple bimodule. For the inductive step, suppose that the conditions of (1) are satisfied but that its conclusion is false, and that $1<s=\operatorname{rank}\left(M_{R}\right)$ is the least integer for which that failure occurs. Let $0 \leq N={ }_{S} N_{R}<{ }_{S} M_{R}$ be such that ${ }_{S}(M / N)_{R}$ is simple. If $N=0$ the conclusions at (1) are clear, so we must have $0 \neq N$. But, by Lemmas 3.1 and 3.3, $N$ must be right inessential in $M$ since $(M / N)_{R}$ is faithful. If $N$ is not biessential in $M$ then there exists a simple sub-bimodule $L \leq M$ such that ${ }_{S} M_{R}={ }_{S} N_{R} \oplus{ }_{S} L_{R}$, and it is then easy to check that both $N$ and $L$ satisfy the hypotheses of (1) and that $\operatorname{rank}\left(N_{R}\right)<s$, so that $\operatorname{BS}(M)=\operatorname{BS}(N) \oplus L$, and $\operatorname{BS}(M)$ is then easily seen to be both $\mathrm{I}(R)$-anti-torsion and bi-essential in $M$, contradicting the assumption about $s$.

So we may suppose that ${ }_{S} N_{R} \leq_{e}{ }_{S} M_{R}$; since each bi-uniform sub-bimodule of $N$ contains a non-zero $\mathrm{I}(R)$-anti-torsion sub-bimodule, and $\operatorname{rank}\left({ }_{S} M_{R}\right)<\infty$, there exists $0 \neq V={ }_{S} V_{R} \leq_{e}{ }_{S} N_{R}$ such that $V_{R}$ is $\mathrm{I}(R)$-anti-torsion and $\operatorname{rank}\left({ }_{S} V_{R}\right)<\infty$. But $\operatorname{rank}\left(V_{R}\right) \leq \operatorname{rank}\left(N_{R}\right)<s$, so by the minimality of $s, \mathrm{BS}(V) \leq_{e} V_{R}$, and $\mathrm{BS}(V)$ is $\mathrm{I}(R)$-anti-torsion. By transitivity (twice) $\mathrm{BS}(V)=\mathrm{BS}(M) \leq_{e} M_{R}$; contradiction. Hence no such $s \in \mathbb{N}$ exists, i.e. the conclusions of (1) are true.

Now suppose that the conditions of (2) are also satisfied. If every $L={ }_{S} L_{R} \leq{ }_{S} M_{R}$ is $\mathrm{I}(R)$-anti-torsion then (Lemma 3.3) $\mathrm{L} / \mathrm{K}$ satisfies (1) whenever $0 \leq K={ }_{S} K_{R}<L={ }_{S} L_{R} \leq{ }_{S} M_{R}$, and it follows easily that the bimodule socle series $0=\mathrm{BS}_{0}(M)<\mathrm{BS}_{1}(M)<\mathrm{BS}_{2}(M)<\ldots<\mathrm{BS}_{q}(M)<\ldots$ terminates at $\mathrm{BS}_{s}(M)=M$ for some $s \in \mathbb{N}$. Each bimodule semi-simple factor $\mathrm{BS}_{r+1}(M) / \mathrm{BS}_{r}(M)$ is bi-noetherian hence also bi-artinian, whence so also is $M$. Alternatively, note that whenever $0 \leq{ }_{S} K_{R}<{ }_{S} L_{R} \leq M$ then $(L / K)_{R}$ is faithful, so that $\operatorname{rank}\left(K_{R}\right)<\operatorname{rank}\left(L_{R}\right)$ by Lemma 3.1. It follows that any strictly descending chain of sub-bimodules of $M$ must be finite.

Remarks 3.5. (A) All the conditions of Lemmas 3.3 and 3.4(1) and 3.4(2) are trivially satisfied when $R$ is a simple ring.

(B) If $R$ is a quasi-primitive non-simple noetherian ring then ${ }_{R} R_{R}$ satisfies condition (1) of Lemma 3.4 but not condition (2). Stafford's example [16] of a quasiprimitive noetherian ring which is not bi-artinian shows that condition (1) alone does not guarantee the existence of a bimodule composition series.

(C) The alternative proof of Lemma 3.4(2) shows that length $\left({ }_{S} M_{R}\right) \leq$ $\operatorname{rank}\left(M_{R}\right)$.

(D) Let $K$ be a commutative field with a non-zero derivation $\delta$, and $n \in \mathbb{N}$ : put $M={ }_{K} M_{K}$ by defining $M_{K}=v_{0} K \oplus v_{1} K \oplus \ldots v_{n} K, y v_{0}=v_{0} y$ and $x v_{j}=v_{j} x+v_{j-1}(x \delta)$ for $0<j \leq n$ and $x, y \in K$ : then ${ }_{K} M_{K}$ is a serial bi-module with composition length $n+1$, while ${ }_{K} M$ and $M_{K}$ are semi-simple $K$-modules (also of length $n+1$ ), and ${ }_{K} M_{K}$ satisfies all the conditions of Lemma 3.4(1) and 3.4(2).

Lemma 3.6. Let $M={ }_{S} M_{R}$ be semi-noetherian and fully of finite right rank, with $R$ quasi-primitive with heart $H$, and suppose that $M_{R}$ is $\mathrm{I}(R)$-torsionfree. Then $\mathrm{BS}(M H)=\mathrm{BS}(M) \leq_{e} M_{R}$. 
Proof. Observe first that, since $M_{R}$ is $\mathrm{I}(R)$-torsionfree, $0 \neq L H \leq M H$ for any $0 \neq L \leq M_{R}$, so $M H \leq_{e} M_{R}$. If $0 \neq I \in \mathrm{I}(R)$ then $(M H) I=M(H I)=M H$, i.e. $(M H)_{R}$ is $\mathrm{I}(R)$-anti-torsion. By Lemma 3.4(1) it suffices to show that, if $0<K={ }_{S} K_{R} \leq{ }_{S} M_{R}$ then there exists $0<{ }_{S} J_{R} \leq{ }_{S} K_{R}$ such that $J_{R}$ is $\mathrm{I}(R)$-anti-torsion; set $J=K H$.

Lemma 3.7. Let $M={ }_{S} M_{R}$ be semi-noetherian and fully of finite right rank, and $R$ be bi-noetherian, If $B$-spec $(M R) \subseteq$ quas $(R)$ then ${ }_{S} M_{R}$ is bi-artinian.

Proof. Set $M_{0}=0$, let $P$ be a maximal right annihilator for $M$, and suppose $0<N={ }_{S} N_{R} \subseteq \operatorname{ann}_{M}(P)$, so $P=\operatorname{ann}\left(N_{R}\right)$ by maximality. By Lemma $3.1 N_{R / P}$ is $\mathrm{I}(R / P)$-torsionfree. If $P<I \in \mathrm{I}(R)$ and $I / P=H=\mathrm{H}(R / P)$ then $0 \neq M_{1}=$ $\mathrm{BS}\left(S(N H)_{R / P}\right) \leq_{e} S(N H)_{R / P}$ by Lemma 3.6, and clearly has finite bimodule length. Either $M_{1}=M$ or, iterating, we can construct a sequence of sub-bimodules $0=M_{0}<M_{1}<\ldots$ such that each factor $M_{i+1} / M_{i}$ has finite bimodule length. Since $M$ is bi-noetherian, the sequence terminates, and $M$ itself has finite bimodule length.

EXAmPle 3.8. The assumption that $M$ is left semi-noetherian cannot be dropped from Lemma 3.7. Let $S$ be a simple noetherian ring which is not artinian, and let $K$ be the centre of S; set $R=\left(\begin{array}{cc}K & S \\ 0 & S\end{array}\right)$ and $M={ }_{R} R_{R}$. Then $R$ is right noetherian but not left semi-noetherian and quas $(R)=\operatorname{spec}(R)$ but $R$ is not bi-artinian.

Lemma 3.9. If $R$ is a bi-artinian ring then $\operatorname{spec}(R)=$ quas $(R)$.

Proof. We may assume that $R$ is prime, and by the bi-d.c.c. has a simple ideal $H$. Since $R$ is prime, $H$ is idempotent and bi-essential.

\section{Main Theorem.}

THeOREM 4.1. A semi-noetherian fully right Goldie ring is bi-artinian if and only if every prime ideal is quasi-primitive.

Proof. Lemmas 3.1, 3.7 and 3.9.

COROLlary 4.2. Let $R$ be a semi-noetherian fully right Goldie ring; then $R$ is biartinian if and only if $R / P(R)$ is bi-artinian.

Remarks 4.3. (A) It was already known [21, p. 217] that, if $M={ }_{S} M_{R}$ is leftright noetherian and $R$ is simple then $M$ has a bimodule composition series, and that a noetherian ring $R$ in which $\operatorname{spec}(R)=\max (R)$ is bi-artinian. A formally narrower result which easily yields this latter conclusion can be found at [3, top of p. 9].

(B) From Example 3.8 it is clear that a ring $R$ must satisfy some sort of twosided finiteness condition in order for the equality $\operatorname{spec}(R)=$ quas $(R)$ to imply that $R$ is bi-artinian (the reverse implication being automatic: Lemma 3.9). It is well known that, if $K$ is a field and $S$ is the direct product of countably infinitely many copies of $K$, then $\operatorname{spec}(S)=\operatorname{quas}(S)=\max (S)$ but $S$ is neither bi-noetherian or 
bi-artinian. Thus the condition that every principal (two-sided) ideal is left and right finitely generated is not sufficient to ensure that, if $\operatorname{spec}(S)=\operatorname{quas}(S)$, then $S$ must be bi-artinian. It would be interesting to know whether the condition that $S$ is leftright semi-noetherian is sufficient.

(C) Theorem 4.1 does not extend to right noetherian rings: see Example 3.8.

(D) It is straightforward to construct non-trivial examples of left semi-noetherian right noetherian rings which are not left noetherian, to which the test in Theorem 4.1 can then be applied. For example, if $A_{1}(-)$ denotes the first Weyl algebra (with the usual generators), if $S$ is a simple ring of characteristic 0 , and if $S$ is right but not left noetherian, then $S$ is certainly left semi-noetherian, and the ring $R=$ $S \oplus X A_{1}(S) \subseteq A_{1}(S)$ can be shown (see [23]) to be left seminoetherian right noetherian but not left noetherian, and to have a bimodule composition series of length 2: see also Section 7.

5. Direct sum decompositions. The proof of the following result uses entirely standard techniques, and is omitted.

THeOREM 5.1. Let $R$ be a ring which is both bi-noetherian and bi-artinian, and let $p>0$ be a rational prime. Then:

(1) the set $T_{p}=\left\{r \in R: \exists n \in \mathbb{N}, r p^{n}=0\right\}$ is an ideal of $R$;

(2) there exists $m=m(p) \in N$ such that $\left(T_{p}\right) p^{m}=0$ and $R=\left(R p^{m}\right) \oplus T_{p}$ (as rings);

(3) $R$ is a finite direct sum of indecomposable bi-noetherian bi-artinian rings each of which is either a $\mathbb{Q}$-algebra or an algebra over $\mathbb{Z} / q^{s} \mathbb{Z}$ for some rational prime $q>0$ and some $s \in \mathbb{N}$.

6. The restricted bi-d.c.c. A ring $R$ is said to satisfy the restricted bi-d.c.c. if $R / I$ is bi-artinian for every $0 \neq I \in \mathrm{I}(R)$, but $R$ itself is not bi-artinian. It is well known that, if $R$ is either noetherian commutative or BPN [19, p. 94] then $R$ satisfies the restricted bi-d.c.c. if and only if $R$ is prime non-bi-artinian and $\operatorname{spec}(R) \backslash\{0\}=\max (R)$. The following is the natural analogue of those results in the present context.

THEOREM 6.1. Let $R$ be a semi-noetherian fully right Goldie ring. Then $R$ satisfies the restricted bi-d.c.c if and only if $R$ is prime non-bi-artinian and $\operatorname{spec}(R) \backslash\{0\}=$ quas $(R)$.

Proof. Suppose $R$ is prime non-bi-artinian and quas $(R)=\operatorname{spec}(R) \backslash\{0\}$. Let $0 \neq I \in \mathrm{I}(R)$; then $\operatorname{spec}(R / I)=$ quas $(R / I)$, so $R / I$ is bi-artinian by Theorem 4.1.

Conversely, suppose $R$ is not bi-artinian but $R / I$ is bi-artinian for each $0 \neq I \in \mathrm{I}(R)$. By Corollary 4.2, $P=\mathrm{P}(R)=0$, i.e. $R$ is semiprime. If $R$ is not prime then $\operatorname{card}(\min (R))=n>1$ and if $Q \in \min (R)$ then $0 \neq Q$, so that $R / Q$ is bi-artinian. Hence $\operatorname{spec}(R / Q)=$ quas $(R / Q)$, and since $Q \in \min (R)$ is $\operatorname{arbitrary,~} \operatorname{spec}(R)=$ quas $(R)$, so that $R$ is bi-artinian; contradiction.

We say that a ring $R$ satisfies the E-restricted bi-d.c.c. if $R$ is not bi-artinian but $R / E$ is bi-artinian for all $E \in \mathrm{E}(R)$. For such rings there is a (less complete) "biessential" analogue of Theorem 6.1. 
THeOREM 6.2. Let $R$ be a semi-noetherian fully right Goldie ring: of the following conditions, (1) implies (2A) while (2A) and (2B) are equivalent:

(1) $R$ satisfies the E-restricted bi-d.c.c.;

(2A) $R / \mathrm{P}(R)$ satisfies the E-restricted bi-d.c.c.;

(2B) $\operatorname{spec}(R) \neq$ quas $(R)$ but $\operatorname{spec}(R) \backslash \min (R) \subseteq$ quas $(R)$.

When condition (2A) (or (1)) is satisfied, $\min (R) \backslash$ quas $(R) \neq \varnothing$.

Proof. Suppose (1) is satisfied, and let $V \in \mathrm{I}(R)$ be such that $P=\mathrm{P}(R)<V$ and ${ }_{R}(V / P)_{R} \leq_{e} R(R / P)_{R}$. Now $P$ is nilpotent, so $X=\operatorname{ann}\left(P_{R}\right)$ is left essential [6, p. 62], hence bi-essential, so $R / X$ is bi-artninian and $\operatorname{spec}(R / X)=$ quas $(R / X)$. It follows by Lemma 3.7 that ${ }_{R} P_{R}$ is bi-artinian. If $P \in \mathrm{E}(R)$ then $R / P$ is bi-artinian, so $R$ is too (Corollary 4.2): contradiction. Let $B=\mathrm{BS}(P)$; then $B \leq_{e}{ }_{R} P_{R}$ since $P$ is bi-artinian, so there exists $0 \neq A \in \mathrm{I}(R)$ such that $A \cap B=0$ and $A \oplus B \in \mathrm{E}(R)$; it is clear that $A \cap P=0$, so $A$ embeds in $R / P$ under the natural map. Let $C \in \mathrm{I}(R)$ be such that $P \leq C$ and $C / P$ is a complement for $(A+P) / P$ in $R / P$. Then $C(A+P)+$ $(P+A) C \leq P$, so $C A+A C \leq A \cap P=0$. Hence $(C / P)(A+P)=0$, and since $A+P \in \mathrm{E}(R)$ it follows successively that $R /(A+P)$ is bi-artinian and that $C / P$ is biartinian (whence $C$ is bi-artinian too). Let $D \in \mathrm{I}(R)$ be such that $P \leq D$ and $D / P=\mathrm{BS}(C / P)$; then $A+P \leq A+D \in \mathrm{E}(R)$ so $R /(A+D)$ is bi-artinian.

Suppose $a \in A$ and $V a=0$; then $V(a+P) \leq P$, but it is standard (since $R / P$ is semiprime) that $\operatorname{ann}_{R / P}\left((V / P)_{R / P}\right)=P / P$; hence $a \in P$. But $A \cap P=0$ so $a=0$. Standard arguments then show that $V A \leq_{e} A_{R}$ so (replacing A by VA in the previous argument) $R /(V A+D)$ is bi-artinian. But $P<V$ and $D / P \leq V / P$, so $V A+D \leq V$, and therefore $R / V$ is bi-artinian, i.e. (2A) is satisfied.

Suppose $(2 \mathrm{~A})$ is satisfied. We may assume that $R$ is semiprime and that quas $(R) \subset \operatorname{spec}(R)$; suppose that $Q \in \operatorname{spec}(R)$ is non-minimal. By standard fact (5) in Section 2, $Q \in \mathrm{E}(R)$, so $R / Q$ is bi-artinian, and hence $Q \in$ quas $(R)$. Suppose (2B) is satisfied, that $R$ is semiprime, and that $I \in \mathrm{E}(R)$. If $R / I$ is not bi-artinian then there exists $J \in \mathrm{E}(R)$ maximal with respect to the properties $I \leq J$ and $R / J$ is not biartinian, and clearly $J \neq R$. But then $R / J$ satisfies the restricted bi-d.c.c., so $J \in \operatorname{spec}(R)$. But $I \leq J$ and $I \in \mathrm{E}(R)$ so $J$ is non-minimal; contradiction. Thus (2B) implies (2A). The final assertion is clear.

Suppose that $R$ is left semi-noetherian and $0 \neq I \in \mathrm{I}(R)$, with $n \in \mathbb{N}$ minimal with respect to the existence of $x_{1}, \ldots, x_{n} \in I$ such that $I=R x_{1}+\ldots+R x_{n}$, and by Zorn's Lemma let $M={ }_{R} M<{ }_{R} I$ be maximal with respect to $R x_{1}+\ldots+R x_{n-1} \leq$ ${ }_{R} M<{ }_{R} I$; then ${ }_{R}(I / M)$ is simple so $\operatorname{ann}\left({ }_{R}(I / M)\right)$ is left primitive. It follows easily that, if $Q \in$ quas $(R)$, then $Q$ is left primitive, and hence that $\mathrm{J}(R) \leq \mathrm{A}(R)$. This means that noetherian rings which satisfy the E-restricted bi-d.c.c. have other pleasing properties.

COROLlaRY 6.3. Let $R$ be a semi-noetherian fully right Goldie ring which satisfies the E-restricted bi-d.c.c, and let $J=\mathrm{J}(R) \leq A=\mathrm{A}(R)$. Suppose that either (1) $R$ is semiprime, or (2) $R$ is fully left Goldie; then $\bigcap_{n \in \mathbb{N}} J^{n}=\bigcap_{n \in \mathbb{N}} A^{n}=0$.

Proof. Let $A^{\prime}=\bigcap_{n=1}^{\infty} A^{n}$ : we know from an earlier remark that $J \leq A$. Let $B=\mathrm{BS}\left({ }_{R} R_{R}\right)=\bigcap_{E \in \mathrm{E}(R)} \stackrel{n=1}{E}$ and let $c \in R \backslash B$. There exists $K(c) \in \mathrm{E}(R)$ such that $c \notin K(c)$. Since $R / K(c)$ has a bi-module composition series, $\operatorname{spec}(R / K(c))=$ quas $(R / K(c))$ by 
Theorem 4.1, so there exist $Q_{1}, \ldots, Q_{s} \in$ quas $(R)$ such that $Q_{1} Q_{2} \ldots Q_{s} \leq K(c) \leq$ $Q_{1} \cap \ldots \cap Q_{s}$; hence $A^{s} \leq K(c)$. It follows that $A^{\prime} \leq B$. If $B=0$ there is nothing further to prove; if $B \neq 0$ let $B^{\prime} \in \mathrm{I}(R)$ be simple, so that $B^{\prime} \leq B$.

In case (1), since $R$ is semiprime $B^{\prime}$ is idempotent, the complement $Q^{\prime}$ of $B^{\prime}$ is quasi-primitive, and $Q^{\prime}=\operatorname{ann}\left(B_{R}^{\prime}\right)=\operatorname{ann}\left({ }_{R} B^{\prime}\right)$. It follows that $Q=\operatorname{ann}\left(B_{R}\right)=$ $\operatorname{ann}\left({ }_{R} B\right)$ is a finite intersection of elements of quas $(R)$, and that $A^{\prime} \leq Q \cap B=0$.

In case (2), if $B \in \mathrm{E}(R)$ then $R / B$ has a bimodule composition series, and hence so does $R$; contradiction. So we may suppose that there exists $0 \neq C \in \mathrm{I}(R)$ such that $B \oplus C \in \mathrm{E}(R)$, and then by hypothesis $R /(B \oplus C)$ is bi-artinian; let $X$ be any term of its bimodule composition series. Copying the proofs of [7, Lemma 5.2] or [8, Lemma 5.2.16] $r$.ann $(X) \in$ quas $(R)$, so $A^{t} \leq B \oplus C$ for some $t \in \mathbb{N}$. By the same arguments again, $B A=0 \leq C$ so $A^{\prime} \leq A^{t+1} \leq C$, and hence $A^{\prime} \leq B \cap C=0$.

REMARKS 6.4. (A) The ring of $2 \times 2$ upper triangular matrices over $\mathbb{Z}$ is a counter-example to the equivalence of (2B) and (1) in Theorem 6.2.

(B) If $R$ is a noetherian ring which satisfies the second layer condition (see [6, p. 183] or [11, p. 188] or [3, p. 4] for details) then quas $(R)=\max (R)($ see $[6, E x .12 \mathrm{E}])$. In that case Jategaonkar showed (see [6. Theorem 12.8]) that $\bigcap_{n \in \mathbb{N}} \mathrm{B}(R)^{n}=0$, a strong version of the Jacobson Conjecture. It is trivial that, in a bi-artinian noetherian ring, the Jacobson and anti-simple radicals coincide with the prime radical, which is nilpotent, but Corollary 6.3 systematically provides non-bi-artinian rings $S$ that do not satisfy the right of left second layer conditions but which do satisfy the Jacobson Conjecture, in the strong version: $\bigcap_{n \in \mathbb{N}} \mathrm{A}(S)^{n}=0$.

ExAmple 6.5. A noetherian ring $S$ which does not satisfy the second layer condition but does satisfy the (strong form of the) Jacobson Conjecture. Let $R$ be a biartinian noetherian ring such that $\max (R) \subset$ quas $(R)$, and put $S=R[X] / \mathrm{B}(R) X R[X]$; it is not difficult to check (see Remark 7.1(A)) that $S$ is noetherian and satisfies the E-restricted bi-d.c.c. but does not satisfy the right or left second layer conditions. We note that $S$ is semiprime if and only if $R$ is semiprime.

\section{Remarks and examples.}

REMARKS 7.1. (A) If $R$ is any bi-artinian ring, it is easy to see (by looking at minimal intersections) that any subset $\varnothing \neq X \subseteq \operatorname{spec}(R)$ whose elements are incomparable must be finite. If $R$ is also bi-noetherian, it follows (by induction on bi-module length) that $\operatorname{spec}(R)$ is finite, and hence that the set of semiprime ideals of $R$ is also finite.

(B) If $S$ is the local commutative noetherian $K$-algebra $K\left[X, Y: X^{2}=X Y=\right.$ $Y^{2}=0$ ], and $\mathrm{K}$ is an infinite field, then $\mathrm{S}$ is (bi-)artinian, but $\mathrm{I}(\mathrm{S})$ is infinite.

(C) See Example 7.2(D) for a bi-artinian noetherian domain with infinitely many ideals.

(D) There is an example, due to Stafford [16], of a non-bi-artinian quasiprimitive noetherian ring $R$ with heart $H$ : the bimodule ${ }_{R} H_{R}$ is left-right faithful and noetherian over the noetherian ring $R$, yet $R$ itself is not bi-artinian, so that quas $(R) \subset \operatorname{spec}(R)$. Thus there is no converse to Lemma 3.7: see also Remark 3.5(B). 
(E) It is standard that a quasi-primitive ideal is right primitive if and only if it is left primitive if and only if it is semi-primitive. If $R$ is semi-noetherian on (say) the left then every quasi-primitive ideal is left (and also therefore right) primitive.

(F) Let $H$ be Sasiada's example of a simple idempotent "ring not necessarily with unity" with Jacobson radical $\mathrm{J}(H)=H$; see [17, p. 151]. Then $H$ is an algebra over a field $K$, and in the bi-artinian ring $R=K \oplus H$ the ideal 0 is quasi-primitive but not semi-primitive.

(G) The examples to follow show that non-commutative bi-artinian noetherian rings can be very unlike commutative (bi-)artinian or non-commutative artinian rings.

EXAmples 7.2. Idealisers and related rings. The first three examples below are well-known, while, as far as we know, the ones following are to some extent new. The techniques needed to establish their properties, however, all stem from [14], and are detailed (in a broader context) in [23].

(A) Let $K$ be a field of characteristic 0 , and $S=A_{1}(K)$ with the usual generators: then $R=K+X S$ is a noetherian bi-artinian domain of bimodule length 2 (using [14, Theorem 7.4]).

(B) With $K, S$ as at (A), let $R=K+K X+X^{2} S, P=K X+X^{2} S \in \operatorname{spec}(R)$ and $H=X^{2} S \in \mathrm{I}(R)$ : then $R$ is a noetherian bi-artinian domain of bimodule length 3 (using [14, Theorem 7.4]), and $H=P^{2}<P$.

(C) Let $\mathrm{F}$ be any field (even finite); the idealiser technique used by Robson in [15] yields a bi-artinian noetherian domain of bimodule length 2 with centre $F$.

(D) Let $S=A_{1}(\mathbb{C})$ and $R=\mathbb{R}+X \mathbb{C}+X^{2} S \subset T=\mathbb{C}+X \mathbb{C}+X^{2} S$ : again using [14, Theorem 7.4], $\mathrm{R}$ is a bi-artininan noetherian domain of bimodule length 4 , and ${ }_{R} T_{R}$ is generated by $\{1, i\}$ and has bimodule length 5 . Note that $\mathrm{I}(R)$ is infinite while $\mathrm{I}(T)$ is finite, (compare Remark 7.1(B)).

(E) Let $K$ be a field of characteristic 0 , and $T=A_{n}(K)$ with the usual generators, and put $\mathrm{X}=X_{1} X_{2} \ldots X_{n}$ : the idealiser $S_{n}$ of XT is a bi-artinian noetherian domain of bimodule length $2^{n}=\operatorname{card}\left(\operatorname{spec}\left(S_{n}\right)\right)$, every $P \in \operatorname{spec}\left(S_{n}\right)$ is completely prime, every $I \in \mathrm{I}\left(S_{n}\right)$ is semiprime idempotent, $\max \left(S_{n}\right)=\{M\}$ where $M$ is a height- $n$ prime, and $2^{n}<\operatorname{card}\left(I\left(S_{n}\right)\right)<2^{2^{n}}$. The first few values of $\operatorname{card}\left(\mathrm{I}\left(S_{n}\right)\right)$ are $3(n=1), 6$ $(n=2)$ and $20(n=3)$, but we do not know a general formula.

(F) Let $K$ be a field, and suppose either that $\operatorname{char}(K)=0$ of that $\operatorname{char}(K)=p>0$ and that $K$ is transcendental over $\mathbb{Z} / p \mathbb{Z}$. There are a $K$-algebra $U=U_{n}(K)$, a multiplicative analogue of $A_{n}(K)$ on $2 n$ generators, and $\Theta \in U_{n}(K)$ (defined similarly to $\mathrm{X}$ at $(\mathrm{E})$ ) such that the idealiser $V_{n}(K)$ of $\Theta U$ has the same properties as those listed at (E) for $S_{n}$.

(G) Suppose $D$ is a left semi-noetherian right noetherian bi-artinian ring, and is either a $\mathbb{Q}$-algebra or a $\mathbb{Z} / p \mathbb{Z}$-algebra with a central subfield $K$ that is transcendental over $\mathbb{Z} / p \mathbb{Z}$. The rings $S_{n}(D)$ or $V_{n}(D)$ defined (in the corresponding cases) exactly as in $(\mathrm{E})$ or $(\mathrm{F})$ respectively are also left semi-noetherian right noetherian bi-artinian rings, and are domains, prime rings or semiprime rings respectively if and only if $D$ has the corresponding property.

(H) Let $K$ be a field of characteristic $0, T=A_{1}(K)$ and $n \in \mathbb{N}$. It can be shown that $\left(T / X^{n} T\right)_{T} \cong\left(T_{T}\right)^{n}$, and it is well-known [11, p. 14] that $\operatorname{End}\left(T_{T}\right) \cong K$ and that $S / X^{n} T \cong \operatorname{End}\left(\left(T / X^{n} T\right)_{T}\right)$, where $S$ is the idealiser of $X^{n} T$ : hence $S / X^{n} T \cong M_{n}(K)$, the full $n \times n$ matrix algebra over $K$. Thus if $R=K+X^{n} T \subset S$ it follows (again using [14, Theorem 7.4]) that $R$ and $S$ are both noetherian bi-artinian domains of bimodule length 2 . It is easy to check that $R / X^{n} T$ embeds in $S / X^{n} T$ as (left) scalar 
multiplication: if $D^{\prime}$ is any ring such that $K \subseteq D^{\prime} \subseteq M_{n}(K)$ then, via the identifications just mentioned, the full inverse image $D$ of $D^{\prime}$ in $S$ is a noetherian bi-artinian domain. In particular, the $n \times n$ upper triangular matrices over $K$ are the epimorphic image of such a domain.

EXAmples 7.3. Standard classes of rings.

(A) If $R$ is a noetherian Asano order (including a Dedekind prime ring) then $R$ is bi-artinian if and only if $R$ is simple: see [11, pp. 139-140].

(B) Let $\mathrm{S}$ be a hereditary noetherian prime (HNP) ring: then $S$ is bi-artinian if and only if $S$ is quasi-primitive if and only if $S$ has a unique minimal non-zero idempotent ideal if and only if $S$ is obtained by iterated idealising at isomaximal (see [11, p. 152]) right ideals finitely many times, starting from a simple HNP ring $T$. Conversely, a prime noetherian bi-artinian ring is a HNP ring if and only if it is obtained by such iterated idealising from a simple HNP ring $T$ (use [11, Prop. 5.6.10 and Theorem 5.6.11] together with Theorem 4.1 above).

(C) A noetherian right fully bounded ring is bi-artinian if and only if it is artinian, since a prime right noetherian right bounded ring can have no proper simple ideal.

ExAmples 7.4. Ring extensions. In examples (B) and (C) by a finite subnormalising extension we mean a ring extension $R \subseteq S$ in which there are elements $u_{1}, \ldots, u_{n} \in S$ such that $S=R u_{1}+\ldots R u_{n}$ and $R u_{1}+\ldots+R u_{j}=u_{1} R+\ldots+u_{j} R$ for $1 \leq j \leq n$ : see $[\mathbf{1 8}]$.

(A) If $R$ is a noetherian bi-artinian ring and $R \subseteq S$ is a finite ring extension, i.e. ${ }_{R} S$ and $S_{R}$ are both finitely generated, then ${ }_{R} S_{R}$ is noetherian bi-artinian (Lemma 3.7), so ${ }_{S} S_{S}$ is a noetherian bi-artinian ring. The reverse implication is false [2].

(B) If $R$ is left semi-noetherian right noetherian bi-artinian, and $R \subseteq S$ is a finite subnormalising extension then ${ }_{R} S_{R}$ is left semi-noetherian right noetherian bi-artinian (adapting [19, Proposition 4.1] and using Lemma 3.7). Hence $S$ is a left seminoetherian right noetherian bi-artinian ring.

(C) If $R \subseteq S$ is a finite subnormalising extension and ${ }_{S} S_{S}$ is noetherian bi-artinian then ${ }_{R} R_{R}$ is noetherian bi-artinian (using [18, Theorem 5.2]). One non-trivial instance of a finite subnormalising extension is at Example 7.2(D).

(D) Suppose that $R$ is a prime noetherian bi-artinian ring, and that $Q=Q_{s}(R)$ is the symmetric ring of quotients of $R$, as defined in [12] and [13, Chapter 3]. It is standard that $R \subseteq Q \subseteq W$, where $W$ is the left-right artinian quotient ring of $R$, and from [20, Theorem 3.1(b)] $H \in \mathrm{I}(Q)$, so that $H Q+Q H \leq R$. If $\mathrm{t} \in H \cap C_{R}(0)$ then $t Q+Q t \subseteq R,{ }_{R} Q \cong{ }_{R}(Q t)$ and $(t Q)_{R} \cong Q_{R}$ so that ${ }_{R} Q_{R}$ is a noetherian (and hence bi-artinian) bimodule, and finally $Q$ is a noetherian bi-artinian prime ring. It can be shown that $Q=Q_{s}(Q)$, i.e. that $Q$ is symmetrically closed in the terminology of [12] and [13, Chapter 3], and hence is a maximal order. A non-trivial instance in which $R \subset Q_{s}(R)$ is at Example 7.2(D).

(E) If $R$ is left semi-noetherian right noetherian bi-artinian and $S=R[X ; \alpha]$ for some automorphism $\alpha$ of $R$ then $S$ is left semi-noetherian right noetherian, but never bi-artinian. Its ring of quotients $T=R\left[X, X^{-1} ; \alpha\right]$ is also left semi-noetherian right noetherian, and is bi-artinian provided that $\alpha$ satisfies a complicated criterion in [24]. If there is a central subfield $K$ of $T$ such that $K=K \alpha$ and the restriction of $\left.\alpha\right|_{K}$ has infinite order, and if the lattice $\{I \in \mathrm{I}(R): I=I \alpha\}$ is bi-artinian then $T$ is a left seminoetherian right noetherian bi-artinian ring (via an easy extension of [9, Lemma 1]). The results in this area generalize [10], which gave a criterion for $T$ to be simple. 
(F) Let $D$ be a division ring (skew field) of characteristic 0 , and for $n, r \in \mathbb{N}$ let $A_{n}(D)$ and $A_{n+r}(D)$ be the $n$th and $(n+r)$ th Weyl algebras over $D$, with the obvious embedding $A_{n}(D) \rightarrow A_{n+r}(D)$. Set $A=\bigcup_{n \in \mathbb{N}} A_{n}(D)$ and $R=A[t], t$ a central indeterminate. It is straightforward to show that $R$ is a fully Goldie domain in which every ideal is generated by a central element (so that $R$ is semi-noetherian), but that $R$ is neither right nor left noetherian.

REMARKS 7.5. (A) For a noetherian ring $R$, the implication: "if $\operatorname{spec}(R)=$ quas $(R)$ then $R$ is bi-artinian", is an easy consequence of [7, Proposition $1.2]$, which asserts that every non-zero noetherian bimodule contains a special type of bimodule called a cell, together with [7, Lemma 5.2], which asserts that, for a noetherian cell $C={ }_{S} C_{R}, H(C) \neq 0$ if $C_{R}$ is faithful and $R$ is quasi-primitive. Jategaonkar also remarks (re-discussing the same material: [8, p. 134]) that "not much is known" about (prime) noetherian rings with non-zero heart.

(B) Suppose $B={ }_{S} B_{R}$ is left semi-noetherian right noetherian, that $R$ is right noetherian, and that $Q=R D^{-1}$ is bi-artinian for some right denominator set $D \subset R$. Then $B^{\prime}={ }_{S}\left(B \otimes_{R} Q\right)_{Q}$ is left semi-noetherian right noetherian bi-artinian, and its bimodule composition series can be used (for example as in [21]) to obtain information about $B$. In the study of noetherian bimodules, this can provide an alternative technique to traditional localisation (in the sense of constructing a ring of fractions $S$ of $R$ in which $S / \mathrm{J}(S)$ is artinian), when that technique is unavailable.

(C) The examples above show that, unless a field $F$ is algebraic over $\mathbb{Z} / p \mathbb{Z}$ (" $F$ is absolutely algebraic"). there exists for any $n \in \mathbb{N}$ a bi-artinian noetherian domain with centre $F$ and of length $2^{n}$. We have so far failed to find a similar construction in the case that $F$ is absolutely algebraic.

\section{REFERENCES}

1. M. F. Atiyah and I. G. MacDonald, Introduction to commutative algebra (AddisonWesley, Reading, Mass., 1969).

2. G. M. Bergman, Sfields finitely right-generated over subrings, Comm. Algebra 11 (1983), 1893-1902.

3. K. A. Brown, The representation theory of noetherian rings, Noncommutative rings. S. Montgomery and L. Small (eds.): (Springer Verlag, 1991) pp. 1-25.

4. A. W. Chatters and C. R. Hajarnavis, Rings with chain conditions (Pitman, London, 1980).

5. P. M. Cohn, Algebra Vol. 2, (John Wiley and Sons, Chichester, 1977).

6. K. R. Goodearl and R. B. Warfield Jr. An introduction to noncommutative Noetherian rings (Cambridge Univ. Press, Cambridge, 1989).

7. A. V. Jategaonkar, Noetherian bimodules, primary decomposition and Jacobson's conjecture, J. Algebra 71 (1981), 379-400.

8. A. V. Jategaonkar. Localization in Noetherian rings, London Math. Soc. Lecture Note Series No. 98 (Cambridge University Press, 1986). $79-85$.

9. D. A. Jordan, Primitive skew Laurent polynomial rings, Glasgow Math. J. 19 (1978), 137.

10. D. A. Jordan, Simple skew Laurent polynomial rings, Comm. Algebra 12 (1984), 135-

11. J. C. McConnell and J. C. Robson, Noncommutative Noetherian rings (John Wiley and Sons, Chichester, 1987).

12. D. S. Passman, Computing the symmetric ring of quotients, J. Algebra 105 (1987), $207-235$. 
13. D. S. Passman, Infinite crossed products, Pure and Applied Mathematics Series Vol. 135 (Academic Press, London, 1989). $45-81$.

14. J. C. Robson, Idealizers and hereditary noetherian prime rings, J. Algebra 22 (1972),

15. J. C. Robson, Simple noetherian rings need not have unity elements, Bull. London Math. Soc. 7 (1975), 269-270.

16. J. T. Stafford, On the ideals of a noetherian ring, Trans. Amer. Math. Soc. 289 (1985), $381-392$

17. F. A. Szasz, Radicals of rings (John Wiley and Sons, Chichester, 1981). 230.

18. E. A. Whelan, Finite subnormalizing extensions of rings, J. Algebra 101 (1986), 211-

19. E. A. Whelan, Normalising elements and radicals, I, Bull. Austral. Math. Soc. 39 (1989), 81-106.

20. E. A. Whelan, The symmetric ring of quotients of a primitive ring is primitive, Comm. Algebra 18 (1990), 615-633.

21. E. A. Whelan, Direct sum decompositions of conformal rings, Acta Math. Hungar. 63 (1993), 211-230.

22. E. A. Whelan, Symmetry conditions in ring and module theory, Ph. D. Thesis, University of East Anglia, 1986.

23. E. A. Whelan, Constructing noetherian rings which fail the second layer condition, in preparation.

24. E. A. Whelan, Surjectivity of the ideal extension map $\mathrm{I}(R) \rightarrow \mathrm{I}\left(R\left[X^{-1} ; \alpha\right]\right)$, Comm. Algebra, submitted. 